\title{
Generation and acceleration of high-charge short-electron bunches
}

\author{
M. E. Conde, W. Gai, R. Konecny, X. Li, J. Power, and P. Schoessow \\ Argonne National Laboratory, Argonne, Illinois 60439 \\ N. Barov \\ University of California Los Angeles, Los Angeles, California 90024 \\ (Received 27 February 1998; published 18 August 1998)
}

\begin{abstract}
We report experimental results on the high peak current electron beam generated at the Argonne Wakefield Accelerator facility for wakefield applications. The facility produces bunch charges in the range 15-100 $\mathrm{nC}$ by using a photocathode based $\mathrm{RF}$ electron gun. The energy of the beam is $14 \mathrm{MeV}$. Our measurements show that for bunches of $100 \mathrm{nC}$, the pulse length can be as short as 30 ps. A detailed systematic study of pulse length versus charge is reported, as well as comparisons with numerical simulations. Dark current beam loading in the high field RF gun is also discussed. [S1098-4402(98)00011-1]
\end{abstract}

PACS numbers: 41.75.Lx, 29.27.Ac, 41.85.Ja, 52.40.Mj

\section{INTRODUCTION}

Advanced particle acceleration methods have been a subject of intensive study in recent years, as evidenced by a series of workshops and conferences [1]. One of the prominent schemes is based on electron beam driven wakefields. This method uses an intense short electron beam to excite wakefields in plasmas [2], in slow wave structures such as iris loaded metallic structures [3], or in dielectric loaded waveguides [4]. Other related methods, such as the free-electron laser based [5] and the structure based [6] two beam acceleration schemes, also use an intense electron beam as a driver. Thus, sources of highcharge short-electron pulses are needed to study these advanced acceleration methods. In general, to achieve high gradient acceleration wakefields, electron bunches of 20-100 nC with pulse lengths of 5-30 ps FWHM are needed. These types of electron sources have not been available until recently.

In order to study and demonstrate the wakefield acceleration methods and the two beam acceleration concept, we have designed and constructed a facility called the Argonne Wakefield Accelerator (AWA) [7]. The AWA consists of three major components: (i) a photocathode based RF electron gun capable of producing up to $100 \mathrm{nC}$ (drive gun) followed by two standing wave linac sections for post acceleration [8], (ii) a second photocathode based RF electron gun capable of generating low-charge shortelectron bunches synchronized with the drive bunches (witness gun) [9], and (iii) an experimental section for wakefield measurements in which the witness beam is accelerated by wakefields generated by the drive beam. The AWA facility has been commissioned and is fully operational, although yet to attain its optimal conditions. The drive gun and two linac sections are the subject of this paper. We discuss the AWA drive gun design and report measurements of the drive electron beam properties. We have obtained electron bunches of 20-100 nC with
FWHM of 15-40 ps, which have been used for initial wakefield experiments in both dielectric loaded structures [10] and plasmas [11]. These unprecedented high-charge short-electron bunches, allied with the uniqueness of having two photocathode RF guns with adjustable delay between their beams, make AWA an ideal facility for the study of electron beam driven wakefield acceleration.

\section{AWA ELECTRON GUNS AND LINAC STRUCTURES}

Figure 1 shows a schematic of the AWA layout. The half-cell drive gun was designed to have a high accelerating field $(92 \mathrm{mV} / \mathrm{m}$ at the photocathode surface) to allow the extraction of high-charge electron bunches and to produce a $2 \mathrm{MeV}$ beam with the limited RF power available at the design time (1.5 MW at $1.3 \mathrm{GHz})$ [12]. The high intensity of the accelerating field in the gun permits the generation and acceleration of short-electron bunches, without having to rely on magnetic pulse compression. Magnesium was chosen to be the photocathode material because of its ruggedness and quantum efficiency $\left(5 \times 10^{-4}\right)$. The $2 \mathrm{MeV}$ bunches generated by the drive gun subsequently pass through two standing-wave $\pi / 2$ mode linac tanks (shunt impedance of $21.5 \mathrm{M} \Omega / \mathrm{m}$ ), increasing the beam energy to about $14 \mathrm{MeV}$. The linac structures have large irises $(10.16 \mathrm{~cm}$ diameter $)$ to minimize the generation of wakefields by the propagation of the high-charge drive bunches [13]. The beam is then focused by quadrupoles and bent by three dipoles to allow for the injection of both the drive beam and the witness beam into the wakefield experimental section. The witness gun [9] is a 6-cell standing-wave $\pi / 2$ mode structure that generates $4 \mathrm{MeV}$ bunches of $300 \mathrm{pC}$. Its photocathode material is copper. The witness beam goes through combining optics and then through the wakefield devices (plasma or dielectric structures) trailing the drive beam, thereby probing the wakefields excited by the 


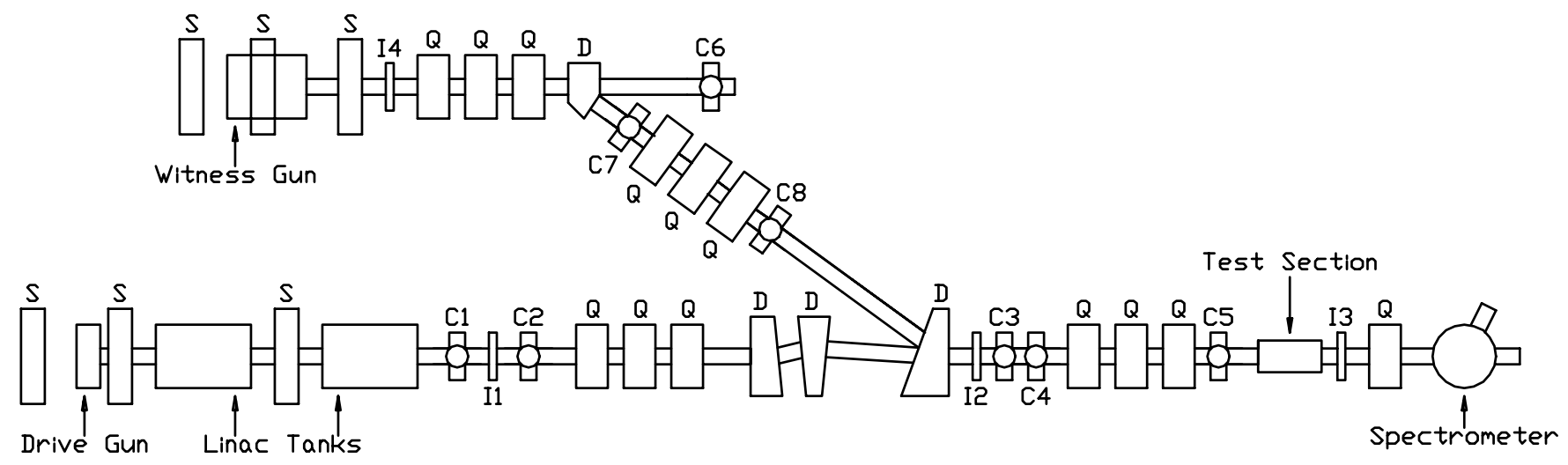

FIG. 1. Schematic of the AWA experimental setup: S, Q, and D indicate solenoids, quadrupoles, and dipoles, respectively; the four integrating current transformers are labeled I1 through I4; the eight diagnostic ports are labeled C1 through C8. Ports C1 and C6 have off axis mirrors that direct the laser beam onto the cathode surface, as well as mirrors that send some of the scattered light off of the cathode to a CCD camera for alignment purposes; all other ports have phosphor targets for electron beam imaging. The aerogel and the pepper-pot plate are located on ports $\mathrm{C} 3$ and $\mathrm{C} 4$, respectively.

drive bunches. Energy changes of the witness beam are measured by a spectrometer magnet located downstream of the wakefield experimental section.

The two RF guns and the two linac structures are powered by a single klystron (Thomson TH2022D; 24 MW, $4 \mu \mathrm{s}$ pulses), via necessary power splitters and phase shifters. The laser system [14] is composed of a dye oscillator $(496 \mathrm{~nm})$ pumped by a tripled yttrium aluminum garnet (YAG), which is then followed by a dye amplifier, a doubling crystal, and finally an excimer amplifier. This laser is capable of producing up to $8 \mathrm{~mJ}$ with $6 \mathrm{ps}$ FWHM at $248 \mathrm{~nm}$. The laser beam is then split and a small fraction of it $(\sim 15 \%)$ sent to the witness gun. There is an adjustable delay between the drive gun and the witness gun laser beams. This allows us to vary the delay between the drive and the witness electron bunches (obviously the RF phases have to be adjusted accordingly, in order to maintain the same launching phase).

\section{DIAGNOSTICS}

There are four integrating current transformers (ICTs) (Bergoz ICT-082-070-20:1) installed on the beam lines to measure bunch charge at various locations, as indicated in Fig. 1. Alternatively, we can use insertable Faraday cups at diagnostic ports along the beam lines and also at the beam dumps. However, the ICTs appear to be more reliable, since they are easier to calibrate, while the Faraday cups suffer from secondary electron emission and electric breakdown due to the high peak voltages generated.

Bunch length measurements are performed using radiation emitted by the electron beam as it passes through a Cerenkov radiator. The Cerenkov light is sent to a Hamamatsu M1952/C1587 streak camera for pulse length measurements. Insertable quartz or aerogel plates are used as Cerenkov radiators. The aerogels require a more elabo- rate holder than the quartz plates, but offer several advantages. The aerogels need to be in a vacuum-tight holder (Fig. 2) which is inserted in the beam path. The electron beam enters our holder through a thin aluminum window $(0.15 \mathrm{~mm})$. It then traverses the aerogel (under atmospheric pressure) and emits Cerenkov radiation, which leaves the aerogel holder through a quartz window. Our aerogels [15] have an index of refraction of 1.009; therefore, the Cerenkov light is emitted at an angle of $7.4^{\circ}$ with respect to the direction of propagation of the $14 \mathrm{MeV}$ electrons. The light is then reflected by a mirror and leaves the vacuum chamber through a diagnostic view port.

Unlike aerogels, quartz plates are compatible with the vacuum of the accelerator and can be easily inserted in the beam path. However, due to their higher index of refraction (1.54), their Cerenkov light is emitted at an angle of $49.5^{\circ}$, making it difficult to collect more than a small fraction of the total cone of radiation. Multiple reflections within the quartz plate are also a problem to be

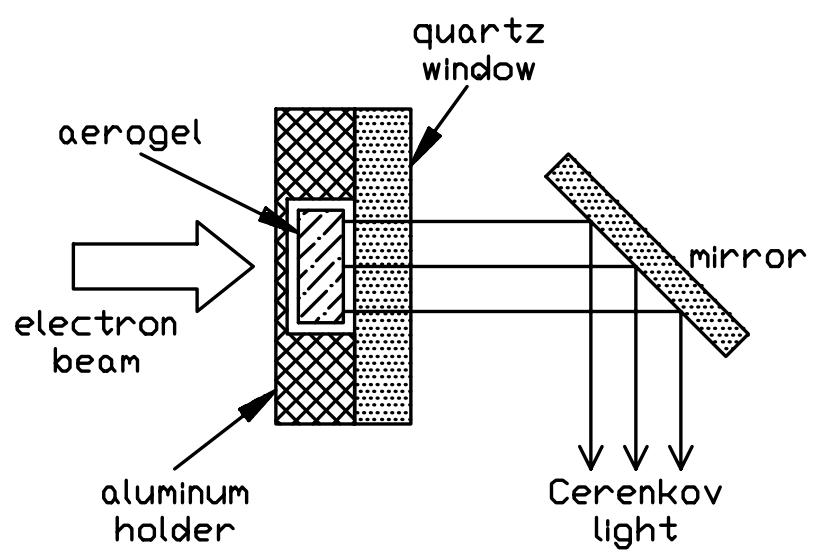

FIG. 2. Schematic of the aerogel holder, indicating the propagation of the electron beam and Cerenkov light. 
considered. They can be minimized by placing the plate such that the radially polarized Cerenkov light leaves the plate at the Brewster angle; however, this introduces a new complication because the electron beam will enter the quartz plate at an oblique angle, introducing a temporal correlation in the transverse profile of the Cerenkov light. Also, in practice, because emission in quartz occurs at large angles, it is harder to achieve good alignment of the associated optics to transport the light to the streak camera.

A pepper-pot plate (indicated in Fig. 1) is used for emittance measurements. It consists of a $1.5 \mathrm{~mm}$ thick tungsten plate with $0.3 \mathrm{~mm}$ diameter holes spaced by $3 \mathrm{~mm}$. A phosphor screen $75 \mathrm{~cm}$ downstream of the pepper-pot plate is used to image the resulting beamlets.

\section{EXPERIMENTAL RESULTS}

Figure 3 shows measurements of pulse lengths as a function of bunch charge. In these plots the charge was
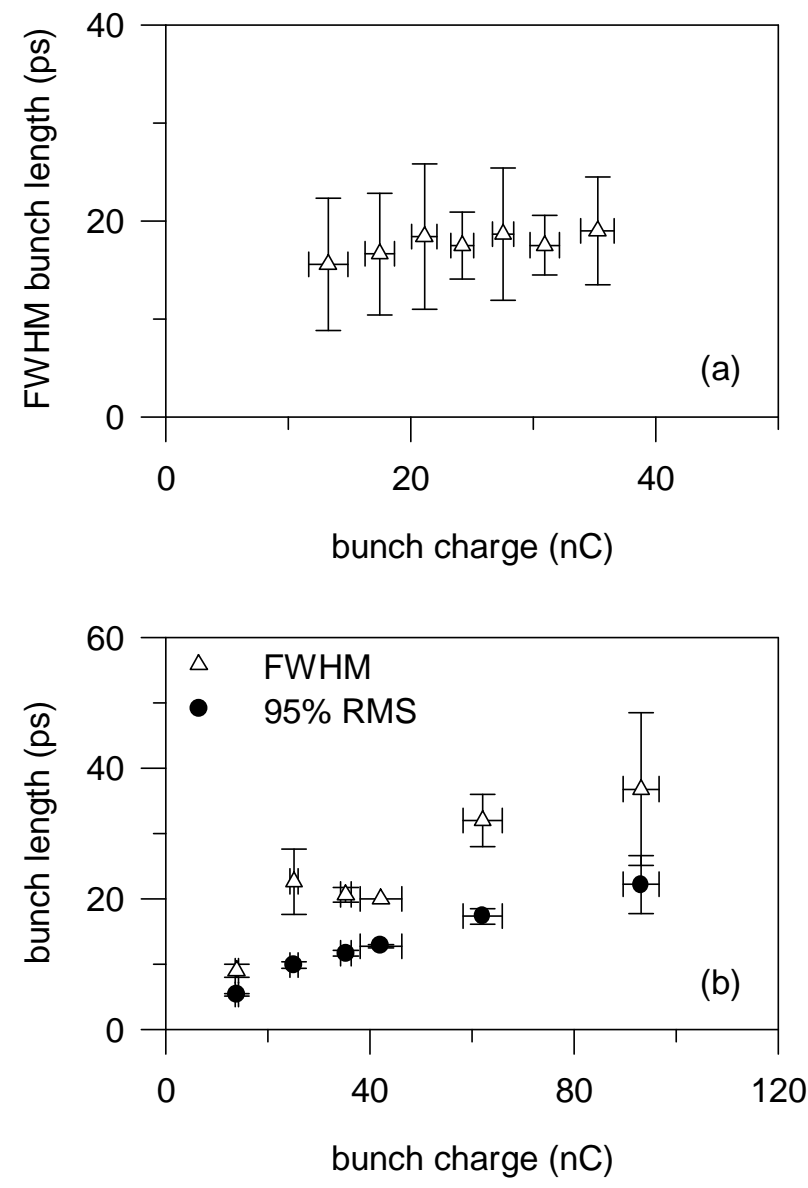

FIG. 3. Measurements of electron bunch length as a function of bunch charge: (a) detailed pulse length measurements in the 15-35 $\mathrm{nC}$ range, each point in the plot is the average of 51 pulses; (b) measurement over a wider range of charges, each point represents the average of three pulses. The error bars in each graph represent the standard deviation. measured by the integrating current transformer I1 (see Fig. 1), and the bunch length was measured using the aerogel radiator and the streak camera. In Fig. 3(a) we show a detailed charge scan in the range $15-35 \mathrm{nC}$ and the corresponding FWHM of the pulses; many pulses were measured in this range and then binned in groups of 41. The plot shows the average value of charge and bunch length for seven bins, indicating that in this charge range the pulse length is almost constant, fluctuating between 15 and 20 ps. The plot in Fig. 3(b) shows a charge scan over a much wider range, but each point in this graph is the average of only three pulses. We have plotted FWHM of the bunch lengths and also the 95\% RMS values (this was calculated taking into account only the section of the pulse profile with intensity within $95 \%$ of the peak value, with the purpose of discarding the effect of the small background noise at the wings of the distribution). The ratio between the 95\% RMS and the FWHM values shows that the pulses are not Gaussian. The large fluctuation in the FWHM of the pulses also shows that the detailed shape of the temporal profile varies considerably from pulse to pulse. Figure 4 shows typical longitudinal profiles for three different values of bunch charge. In all of these bunch length measurements the changes in bunch charge are accomplished by varying the laser pulse energy (either by purposely attenuating the laser beam or due to the natural fluctuations in the laser power); when the change in the emitted bunch charge is large, it becomes necessary to readjust the solenoids and RF phases to compensate for the changes in the space-charge forces.

Emittance measurements of high-current electron beams in this energy range $(14 \mathrm{MeV})$ are quite difficult, because the beam is still space-charge dominated. We attempted to measure the emittance of the drive beam with a pepper-pot plate. Unfortunately, the resulting images are rather irregular. We are now trying to determine if that is caused by scattering on the holes, by nonlinear response of the phosphor screen, or simply by a very irregular distribution of particles in the phase space. It has been suggested that this pepper-pot technique is not really adequate for emittance measurements of beams in our range of parameters [16]. As an alternative, we used the transmission of the electron beam through a small diameter beam pipe of known length to estimate the emittance. By knowing the beta function of the lattice and fitting the waist of the beam as a function of the emittance, we estimated a physical emittance of $20 \mathrm{~mm}$ mrad for a $20 \mathrm{nC}$ beam.

\section{DARK CURRENT BEAM LOADING IN THE DRIVE GUN}

The drive gun generates a large amount of dark current, which we believe to be due to some surface damage that resulted from a disastrous vacuum failure during the initial $\mathrm{RF}$ conditioning of the gun. There is strong evidence of 

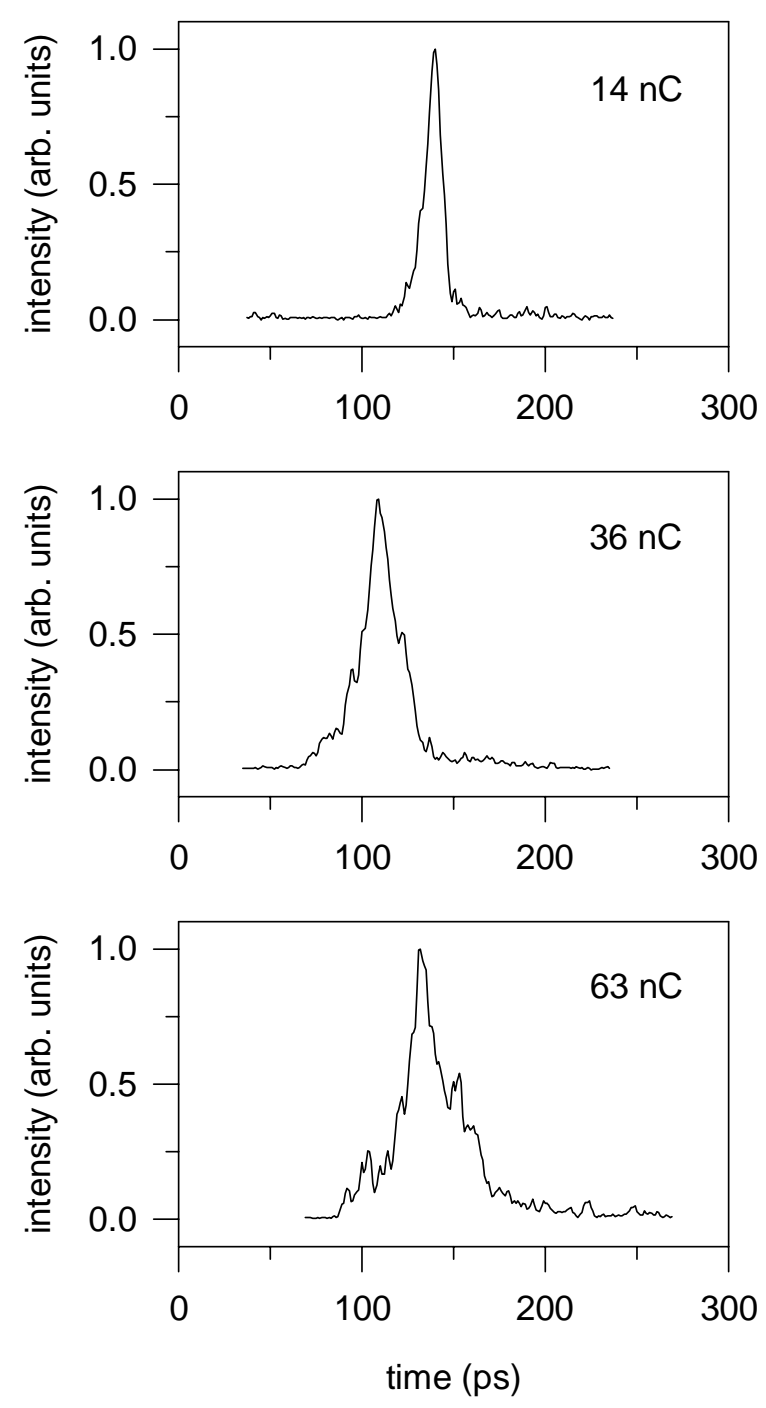

FIG. 4. Longitudinal beam profile for various bunch charges, as measured with a streak camera. The origin of the time scale is arbitrary.

beam loading caused by this large amount of dark current. Figure 5 shows the RF power measured at a waveguide directional coupler located next to the gun. The power coupling into the gun is very good when the gun is driven with up to $1 \mathrm{MW}$ of power, with a negligible amount of $\mathrm{RF}$ being reflected back from the gun. Above that level the coupling deteriorates and a significant fraction of the input power is reflected back.

Another indication of beam loading was observed when we measured the energy of the dark current emitted by the gun (this measurement was conducted with no RF power present in the linac tanks). Figure 6 shows that the energy of the dark current does not scale linearly with the square root of the RF power. Interestingly, it does not scale linearly even if we subtract the fraction of the RF power that is reflected back from the gun. This indicates that not only the dark current changes the coupling into

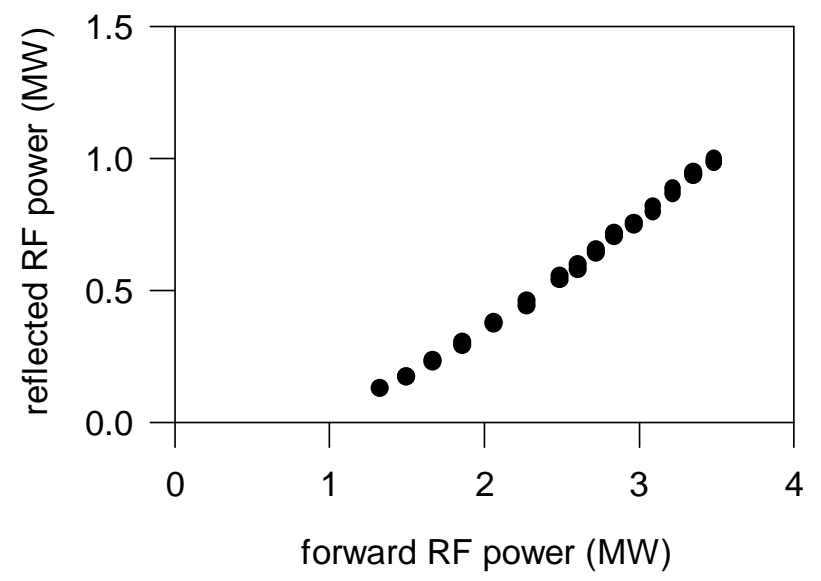

FIG. 5. RF power reflected from the drive gun as a function of the power sent to the gun (forward).

the cavity, but also that a significant fraction of the RF power is taken away by dark current.

Further evidence of the deleterious effect of the dark current was provided by numerical studies of the accelerating field in the drive gun. The modeling of the gun cavity with the code SUPERFISH [17] indicates that an axial electric field in excess of $110 \mathrm{MV} / \mathrm{m}$ should be present on the cathode surface when the gun is driven with $2 \mathrm{MW}$ of RF power. However, when we studied the transport of charge as a function of the gun solenoidal field, we concluded that the experimental measurements can be reproduced by numerical calculations with PARMELA [17] only if the accelerating gradient is reduced to about $55 \mathrm{MV} / \mathrm{m}$, as shown in Fig. 7. In this graph the solenoid located between the two linac tanks was turned off (both in the experiment and in the numerical simulations), and then the charge reaching the integrating current transformer I1 was plotted as a function of the magnetic field generated by

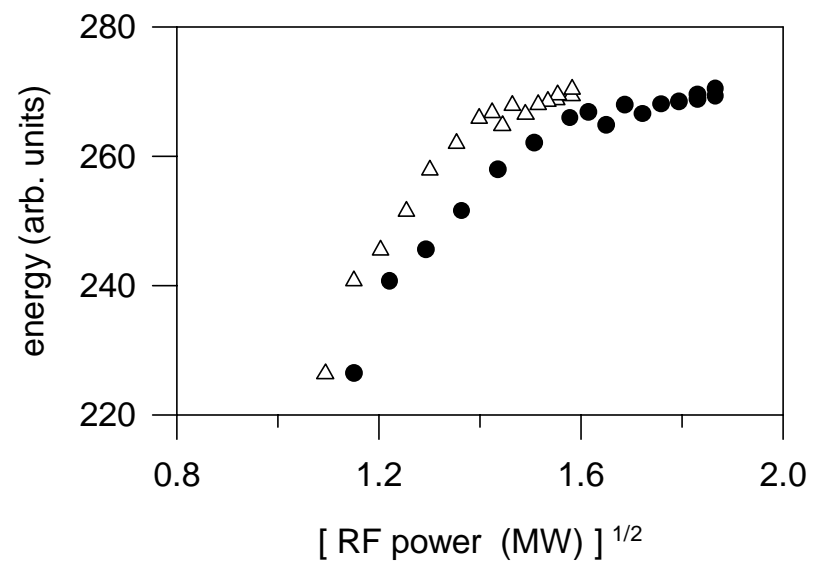

FIG. 6. Dark current energy (centroid of the distribution) as a function of the square root of the input RF power. The circles refer to the forward input power and the triangles correspond to the difference between forward and reflected power. 


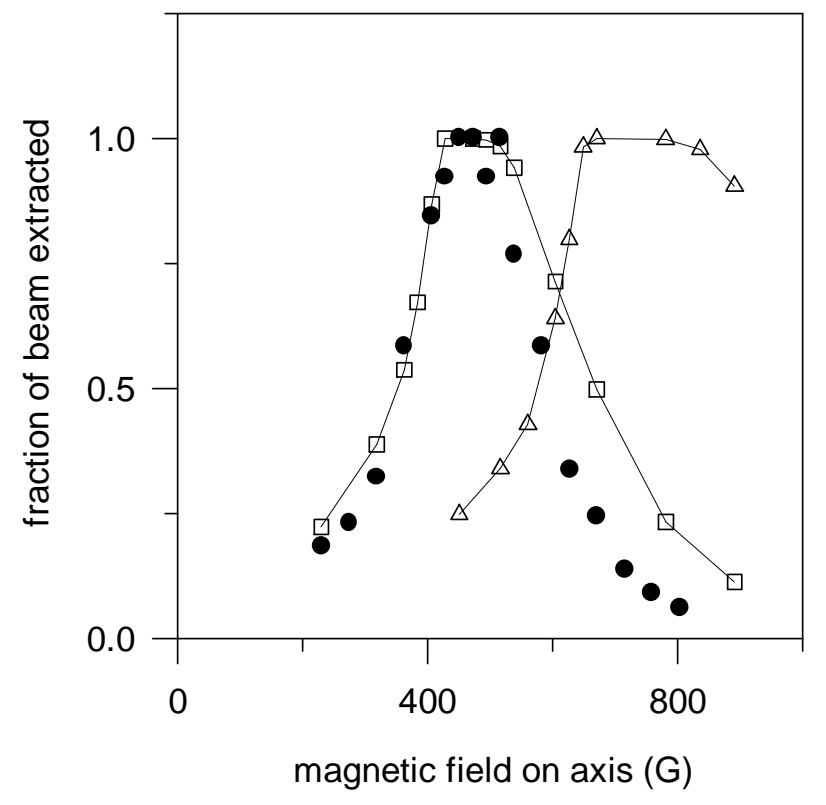

FIG. 7. Fraction of the electron beam charge which is extracted from the drive gun, as a function of the solenoidal field. The points represented by circles correspond to experimental data; the squares and triangles correspond to numerical simulation results using 55 and $92 \mathrm{MV} / \mathrm{m}$, respectively.

the gun solenoid. Clearly, the experimental data are more closely reproduced by simulations employing a lower accelerating gradient.

\section{NUMERICAL SIMULATIONS}

We have executed an extensive set of runs with PARMELA, attempting to understand the implications of the suspected lower gradient in the drive gun. These data are summarized in Fig. 8, which compares the extracted charge, bunch length, and emittance for two different values of accelerating gradient in the gun: the design value of $92 \mathrm{MV} / \mathrm{m}$ and a lower value of $65 \mathrm{MV} / \mathrm{m}$. Every point in these plots has been optimized for best performance (adjusting solenoidal fields and RF pulses). We notice that the amount of charge that can possibly be extracted from the gun and linacs goes down as the accelerating gradient is lowered [Fig. 8(a)]. The emittance of the beam per unit of charge is slightly higher with the lower accelerating gradient [Fig. 8(c)]. The strongest effect of the lower gradient is seen in the predicted bunch length of the pulses [Fig. 8(b)], with an increase as large as $50 \%$ in the bunch length of the highest charge bunches.

\section{DISCUSSION AND SUMMARY}

We are approaching the design goals of the AWA facility $(100 \mathrm{nC}, \sim 30 \mathrm{ps}$ FWHM). There are still many aspects of the generation of these unprecedented high-charge short-electron bunches that call for more detailed studies. While this constitutes an interesting and challenging re-
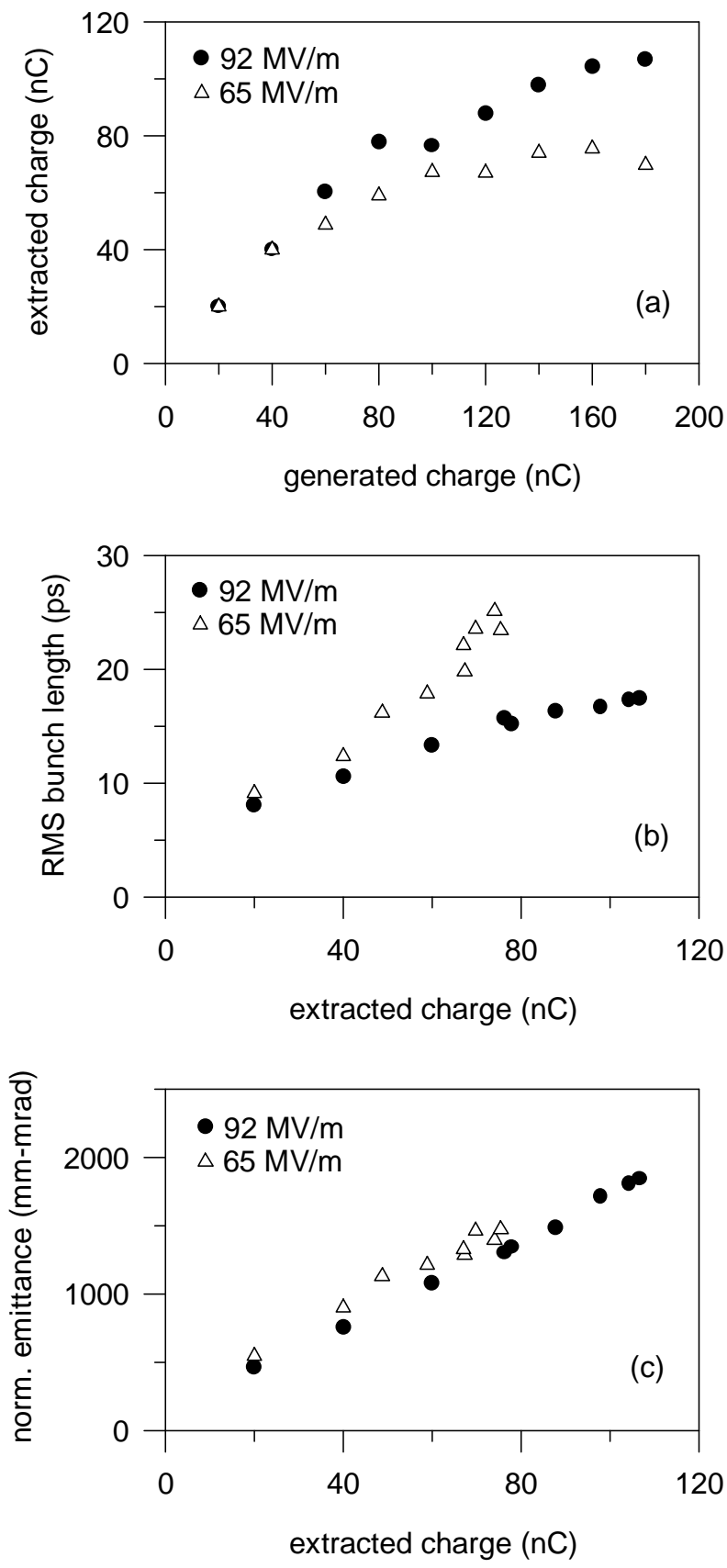

FIG. 8. Numerical simulation results comparing the performance of the electron gun with accelerating gradients of 92 and $65 \mathrm{MV} / \mathrm{m}$ : (a) extracted charge as a function of the charge generated at the cathode surface; (b) RMS bunch length as a function of the extracted charge; (c) normalized emittance as a function of the extracted charge.

search program in itself, we are concomitantly proceeding with experiments on plasmas and dielectric wakefields. We have measured acceleration of the witness beam in dielectric loaded structures [10] and have observed focusing of the electron beam by an underdense plasma [11]. We are presently pursuing wakefield acceleration experiments in underdense plasmas and developing 
new dielectric loaded structures that will employ RF pulse compression to enhance the accelerating gradient.

To further improve the AWA beam quality and, consequently, the performance of the wakefield experiments would require higher accelerating gradients in the drive gun. We believe that the present gradient is considerably lower than the design value due to the excessive dark current, which loads the gun and degrades the RF power coupling. Currently, we are building an identical copy of the present gun in collaboration with the Synchrotron Radiation Research Center (Taiwan). This new gun should provide a much better quality beam, as the simulation results of the previous section indicate.

We have also studied a new gun design with one and a half cells, as an L-band version of the well-known S-band Brookhaven gun [18]. Numerical simulations indicate that $100 \mathrm{nC}$ bunches with $10 \mathrm{ps}$ FWHM are achievable.

In summary, the construction and commissioning of the AWA facility have been completed and it is now fully operational. Although the beam quality has been somewhat hindered by the heavy beam loading due to the excessive dark current, the facility has produced highcharge short-electron bunches, which have been used in wakefield experiments in both dielectric loaded structures and plasmas. We are currently examining possible ways to improve the quality of the electron beam and, at the same time, proceeding with wakefield experiments in dielectrics and plasmas.

\section{ACKNOWLEDGMENTS}

This work is supported by the Department of Energy, Division of High Energy Physics, under Contract No. W31-109-ENG-38.

[1] For example, see Advanced Accelerator Concepts, edited by S. Chattopadyay J. McCullough, and P. Dahl, AIP Conf. Proc. No. 398 (AIP, New York, 1997).

[2] J. B. Rosenzweig, D. B. Cline, B. Cole, H. Figueroa, W. Gai, R. Konecny, J. Norem, P. Schoessow, and J. Simpson, Phys. Rev. Lett. 61, 98 (1988).

[3] H. Figueroa, W. Gai, R. Konecny, J. Norem, A. Ruggiero,
P. Schoessow, and J. Simpson, Phys. Rev. Lett. 60, 2144 (1988).

[4] W. Gai, P. Schoessow, B. Cole, R. Konecny, J. Norem, J. Rosenzweig, and J. Simpson, Phys. Rev. Lett. 61, 2765 (1988).

[5] A. M. Sessler, in The Laser Acceleration of Particles, edited by P. J. Channel, AIP Conf. Proc. No. 91 (AIP, New York, 1982), p. 151.

[6] The CLIC Study Group, in Proceedings of the 1993 Particle Accelerator Conference, Washington, DC, 1993 (AIP, New York, 1993), p. 540.

[7] J. Simpson, Report. No. ANL-HEP-TR-89-81, 1990.

[8] C. H. Ho, Ph.D. thesis, UCLA, 1992 (unpublished).

[9] J. G. Power and M. E. Conde, Rev. Sci. Instrum. 69, 1295 (1998).

[10] P. Schoessow, M. Conde, W. Gai, R. Konecny, J. Power, and J. Simpson, in Proceedings of the 1997 Particle Accelerator Conference, Vancouver, Canada, 1997 (to be published).

[11] N. Barov, M.E. Conde, W. Gai, and J. Rosenzweig, Phys. Rev. Lett. 80, 81 (1998).

[12] W. Gai, C. Ho, R. Konecny, S. Mtingwa, J. Norem, J. Rosenzweig, P. Schoessow, J. Simpson, B. Cole, and M. Rosing, in Proceedings of the 1989 Particle Accelerator Conference, Chicago, IL, 1989 (IEEE, New York, 1989), p. 612.

[13] E. Chojnacki, R. Konecny, M. Rosing, and J. Simpson, in Proceedings of the 1993 Particle Accelerator Conference, Washington, DC, 1993 (IEEE, New York, 1993), p. 815.

[14] W. Gai, R. Konecny, and J. Power, Int. J. Mod. Phys. (Proc. Suppl.) 2A, 522 (1993).

[15] We thank J. Rosenzweig for initiating us in the use of aerogel radiators and J. Oyang and P. Tsou for making them available to us.

[16] R. Sheffield (private communication).

[17] SUPERFISH and PARMELA, Los Alamos National Laboratory Report No. LA-UR-96-1834, 1997; Report No. LA-UR96-1835, 1996.

[18] K. Batchelor, I. Ben-Zvi, R. C. Fernow, J. Fischer, A. S. Fisher, J. Gallardo, Xie Jialin, H. G. Kirk, R. G. Malone, Z. Parsa, R. B. Palmer, T. Rao, J. Rogers, J. Sheehan, T. Y.F. Tsang, S. Ulc, A. van Steenbergen, M. Woodle, R.S. Zhang, I. Bigio, N. Kurnit, T. Shimada, K. T. McDonald, D. P. Russel, Z. Y. Jiang, C. Pellegrini, and X. J. Wang, Proceedings of the 1990 European Particle Accelerator Conference, Nice, France (Editions Frontíers, Gif-sur-Yvette, France, 1990), p. 541. 